\title{
Probing Threading Dislocations in a Micrometer-Thick GaN Film by High-Voltage Scanning Transmission Electron Microscopy
}

\author{
Kazuhisa Sato ${ }^{1 *}$ and Hidehiro Yasuda ${ }^{1}$ \\ 1. Research Center for Ultra-High Voltage Electron Microscopy, Osaka University, Ibaraki, Japan \\ * Corresponding author: sato@uhvem.osaka-u.ac.jp
}

Dislocations in a semiconductor crystal, such as gallium nitride $(\mathrm{GaN})$, degrade its optical and electrical properties. To detect and characterize lattice defects in a thick crystalline specimen which can be regarded as bulk, high-voltage electron microscopy (HVEM) is a powerful technique [1, 2]. The penetration of relativistic electrons depends on imaging conditions as well as materials, and quantitative unified views regarding the maximum usable thickness have not been obtained. Recent interest in crystal defects in semiconductor devices has drawn renewed attention to visualizing microstructures in an extremely thick specimen by HVEM. The authors focused on high-voltage scanning transmission electron microscopy (STEM) as a tool for imaging precipitates and dislocations in micrometer-thick crystals [3, 4]. In this study, we examined quantitative assessment of the maximum usable thickness of GaN crystal with high-density threading dislocations (TDs) using HV-STEM [5].

Wedge-shaped specimens were fabricated from a heteroepitaxial GaN film grown on $\alpha-\mathrm{Al}_{2} \mathrm{O}_{3}(0001)$ using a focused ion beam system. Dislocations in a GaN film were observed using a JEOL JEM1000EES operating at $1 \mathrm{MV}$ equipped with a $\mathrm{LaB}_{6}$ cathode. We subjected a specimen to a two-beam condition by exciting the -1100 or 1-100 reflection of wurtzite GaN. In the bright-field (BF) STEM imaging, the beam convergence was set to a semiangle of $3.75 \mathrm{mrad}$ using a $50-\mu \mathrm{m}$ condenser aperture and the outer collection angle on the BF detector was set to 4-25 mrad. STEM images $1 \mathrm{k} \times 1 \mathrm{k}$ in size were acquired with a dwell time of 256-1365 $\mu$ s/pixel depending on the specimen thickness.

Figure 1(a) shows a BF-STEM image of the wedge-shaped GaN with high-density TDs. The specimen becomes thicker from the left $(0.5 \mu \mathrm{m})$ to the right $(2.5 \mu \mathrm{m})$ of the image. Appearance of the specimen and the selected area electron diffraction (SAED) pattern are shown in Figures 1(b) and 1(c), respectively. TDs are seen as lines almost parallel to [0001] $]_{\mathrm{GaN}}$ with dark contrast in a bright background, and the dislocation images become blurred as the thickness increases. Judging from the invisibility criterion for wurtzite GaN, these TDs in GaN are almost either a-type (threading-edge) or a+c-type (threading-mixed). Then the specimen was turned upside down and comparable observation was made. It was found that the same dislocations were identified irrespective of the observation direction.

Figure 2 shows the thickness dependence of the width of dislocation images measured from the STEM and TEM images. Here, the width is defined as full width at half maximum of the image intensity profile measured perpendicular to a dislocation line. To avoid ambiguity regarding the visibility of dislocations, we introduced a criterion for the evaluation of usable thickness: theoretical dislocation width $\xi_{\text {hkl }} / \pi\left(\xi_{\text {hkl }}\right.$ stands for the extinction distance of the hkl reflection excited for imaging) was employed as the criterion. Dashed line indicates the criterion $\xi_{1-100} / \pi=28.2 \mathrm{~nm}$, and we judged data points below this line are "observable". As seen, width of dislocation images increases as the thickness increases. The obtained maximum usable thickness is $6.9 \mu \mathrm{m}$ for BF-STEM and $4.4 \mu \mathrm{m}$ for BF-TEM operating at 1 MV. HV-STEM is hence the suitable imaging technique that can reveal the microstructures of a 
micrometer-order thick specimen in a nanometer-scale spatial resolution as well as a multi-scale field of view [6].

\section{References}

[1] H. Fujita and T. Tabata, Jpn. J. Appl. Phys. 12 (1973), p.471.

[2] G. Thomas and J. -C. Lacaze, J. Microsc. 97 (1973), 301.

[3] K. Sato et al. Mater. Trans. 57 (2016), 918.

[4] K. Sato, Y. Yamashita, H. Yasuda, and H. Mori, Jpn. J. Appl. Phys. 56 (2017), p.100304.

[5] K. Sato and H. Ysauda, ACS Omega 3 (2018), p.13524.

[6] The authors wish to thank Mr. A. Ohsaki, Mr. Y. Agatsuma, Mr. S. Takakuwa, Dr. S. Ohta, and Mr. M. Ohsaki of JEOL Ltd. for their support using the JEM-1000EES. KS acknowledges Professor Emeritus H. Mori of Osaka University for his invaluable comments. This study was partially supported by JSPS KAKENHI Grant Number JP17H02746.



Figure 1. (a) BF-STEM image of the wedge-shaped GaN with high-density TDs, (b) appearance of the specimen, and (c) SAED pattern.

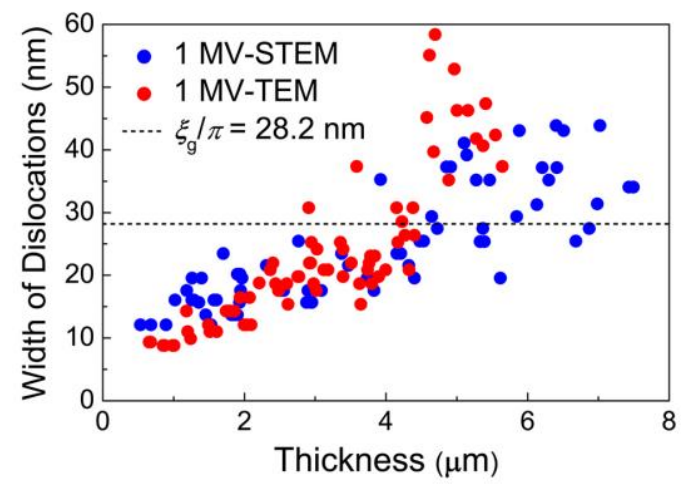

Figure 2. Thickness dependence of the width of the dislocation images measured from STEM and TEM images. 